\title{
Solute transport by suspended buoyant particles
}

\author{
Iván Colecchio ${ }^{1}$, Natalia Arze ${ }^{1}$, Georgina Flores $^{1}$, Ana Quijandria $^{1}$, and Alejandro Boschan, ${ }^{1, *}$ \\ ${ }^{1}$ Grupo de Medios Porosos, Facultad de Ingeniería, Universidad de Buenos Aires, CONICET, Paseo Colón 850, (C1063ACV) \\ Buenos Aires, Argentina.
}

\begin{abstract}
The transport of a colouring solute, driven by the buoyant displacement of microscopic suspended particles, and in the absence of net flow, is studied experimentally in a Hele Shaw cell. Initially, a sharp interface between a transparent fluid without particles and an underlying coloured suspension is obtained. From this situation, the suspended particles rise, carrying the solute in the form of a fingering instability across the interface, where a light transmission technique is used to measure the local solute concentration. This one attains an asymptotic value that increases with the solid fraction $\phi$ of suspended particles, and decreases with the distance to the interface. The solute mass discharge also increases with $\phi$, always being relatively small $(<3 \%)$. The onset and development of the instability as the mechanism driving the transport of the solute is discussed.
\end{abstract}

\section{Introduction}

The flow and sedimentation of suspended granular particles occurs in many natural processes. It is known that the velocity of particles settling in a still fluid fluctuate due to density inhomogeneities, generating spatial structures [1]. Moreover, the presence of suspended particles may trigger several types of instabilities, such as the particle-driven Rayleigh Taylor instability [2], or the settling driven convection instability [3].

On the other hand, the transport and fate of dissolved contaminants (either organic or inorganic solutes) in subsurface and superficial natural flows is of importance in environmental applications such as water quality preservation and waste management.

When suspended particles and a solute coexist, the presence of particles modifies the local constitutive properties (e.g., density, viscosity), which in turn affects significantly the transport of the solute [4]. This issue has been studied numerically and in lab experiments in cylindrical conduits and Hele-Shaw cells, and at different spatial scales, mainly for pressure-driven flows [5-7] with neutrally buoyant particles, or even including the effect of gravitational settling [8].

On the other hand, the transport of passive scalars in the absence of net flow has been mainly studied in geophysics and then mostly in field, natural or numerical experiments [9], which examined particledriven salt or heat [10] exchange between the water column and the sediment bed.

In the lab experiments presented here, we investigate how, in the absence of flow, the presence of buoyant monodisperse spherical particles can drive the transport of solutes over macroscopic distances. The influence of the suspension solid fraction $\phi$ is studied.

\section{Experimental setup and procedure}

The Hele-Shaw cell (set vertically) is composed of two Plexiglas plates $(25 \mathrm{~cm} \times 16 \mathrm{~cm} \times 1.2 \mathrm{~cm})$ screwed together, one has a (rectangular "squircle" shaped) groove in its inner face, in which an O-ring is housed to seal the interior of the cell once the fourteen screws are adjusted. Mylar spacers $(0.25 \mathrm{~mm}$ thick) are placed inbetween the screws to provide a minimum separation between the plates. Low-density thin EVA foam sheets cuttings are placed within the cell to shape its void space as shown in Fig. 1.

The inlets (top-bottom) and outlets (left-right) are controlled by 3 -way valves.

The auxiliary flow system is designed to obtain an initial sharp interface between a transparent fluid without particles (situated in the upper part of the cell) and a coloured particle suspension (situated in the lower part of the cell). The verticality of the cell was controlled carefully, with an error of $0.3 \%$, to avoid Boycott effects.

The transparent fluid (density $=1054.7 \mathrm{~kg} / \mathrm{m}^{3}$, viscosity $=1.79 \mathrm{mPa} . \mathrm{s}$ ) is a mixture of water and glycerol, with a small amount of SDS to avoid particle aggregation, while the suspension carrier fluid (density $=1057.1 \mathrm{~kg} / \mathrm{m}^{3}$, viscosity $=1.86 \mathrm{mPa} . \mathrm{s}$ ) is a similar mixture, but also contains a concentration $\mathrm{C}_{0}=4 \mathrm{~g} / \mathrm{l}$ of a light-absorbing colouring solute (Acid Blue 93). Both fluids are then miscible. The particles are monodisperse spherical polystyrene beads (density $=1.05 \mathrm{~g} / \mathrm{cm}^{3}$, diameter $=40 \mu \mathrm{m})$. Their diameter and circularity are characterized in [11]. 
After vigorous stirring in an external reservoir, the suspension and the transparent fluid are rapidly transferred to both syringes, which are then set in their pumps at the same flow rate, and the auxiliary flow system is started. This way, it was possible to obtain, midway between the two inlets, a sharp and clear initial interface separating the coloured suspension and the transparent fluid.

The initial configuration is stable (denser fluid below). Particles are less dense than both fluids: the choice of rising (and not settling) particles made it possible to use fluids with higher viscosity, which helped stabilizing perturbations of the initial interface during the preparation of the experiment [12].

This condition was critical: a number of tests using the same density for both fluids, particles dispersed in both fluids instead of only in the coloured one, and with the particles settling instead of rising, were performed, but a clear initial interface was impossible to obtain.

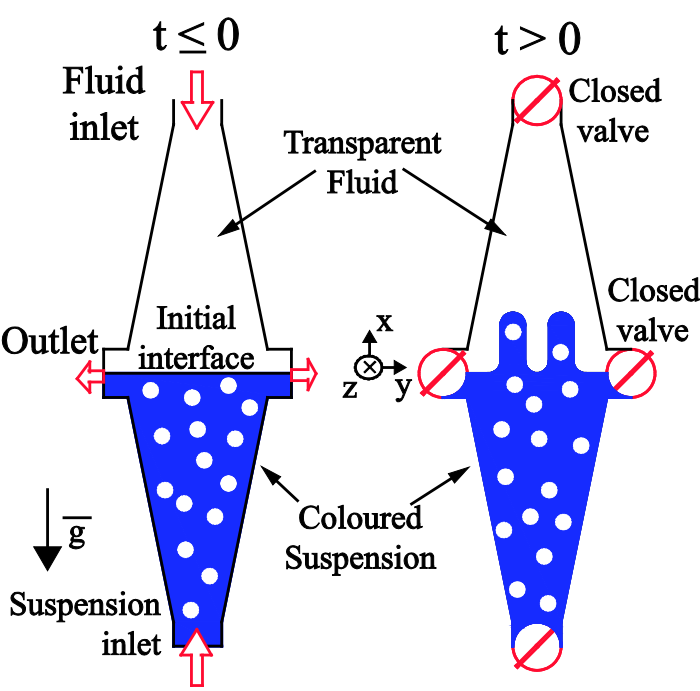

Figure 1. Scheme of the experimental setup. The auxiliary flow system (left, $t<0$ ) makes it possible to obtain a sharp interface as initial condition between the coloured suspension and the transparent fluid. Once the system is stopped and the valves closed (right), the less dense particles rise carrying the solute across the initial interface.

At $t<0$ the transparent fluid injected at moderate flow rate by the first syringe enters the cell through the upper inlet, while the coloured suspension does it through the lower inlet. Both exit the cell through the two lateral outlets.

At $t=0$ the pumps are stopped and the valves are closed, the remaining flow attenuates in few seconds. The interface adjusts slightly into its initial position, in a much smaller timescale than that of the buoyant displacement of the particles.

With the help of a backlight panel with rectified power supply, a CoolSnap cf camera acquired 12-bit grey-level images of the central rectangular zone of the cell in front view, yielnding a spatial resolution of 11.3 pixels $/ \mathrm{mm}$ and a time interval of $180 \mathrm{~s}$ (the Stokes time is $6 \mathrm{~s})$.

\section{Data processing}

The grey-level images of the light intensity transmitted through the cell were transformed into normalized concentration maps $C^{\prime}(x, y) \equiv C(x, y) / C_{0}$ by using a calibration curve obtained by measuring, in a pixel-bypixel basis, the transmitted intensity for a set of pattern concentrations.. The procedure is described in detail in [13]. The relative error in the measurement of the concentration is $1.5 \%$. The solute concentration is assumed to be homogeneous across the cell gap ( $z$ direction). Fig. 2 shows, for different values of $\phi$, the concentration fields at $t=2700 \mathrm{~s}$
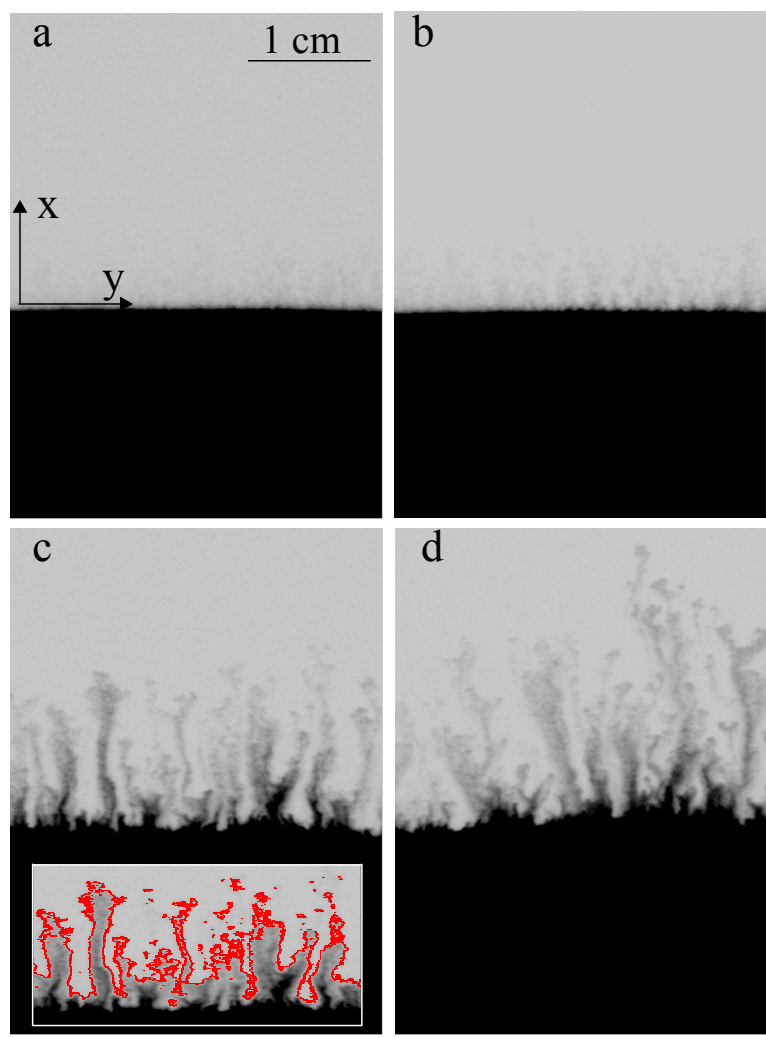

Figure 2. Normalized concentration $C^{\prime}(x, y)$ maps for $\phi=1 \%$ (a), 2\% (b), 3\% (c) and 5\% (d) at $t=2700 \mathrm{~s}$. Fingering is observed in all cases, but much stronger for $\phi \geq 3 \%$. The fingers grow faster, and carry more solute across the initial interface, as $\phi$ increases. Inset in c) depicts the isoconcentration front for $C^{\prime}=0.03$ for the map shown in c). The iso-concentration fronts are used in the following to estimate a characteristic fingertip velocity.

From these maps, spatiotemporal diagrams $C^{\prime}(x, t)$ were generated by averaging the concentration $C^{\prime}(x, y, t)$ in the $y$-direction.

Fig. 3 shows the map of $C^{\prime}(x, t)$ for $\phi=3 \%$, were the three monitoring zones are indicated [14]. These are situated at $1 \mathrm{~mm}\left(x_{1}\right), 2.5 \mathrm{~mm}\left(x_{2}\right)$, and $4 \mathrm{~mm}\left(x_{3}\right)$ respectively higher than the initial position of the interface and, are $0.5 \mathrm{~mm}$ wide.

From the spatiotemporal maps, the variation of the normalized concentration $C$ ' with time at the monitoring positions was obtained (breakthrough curves). 


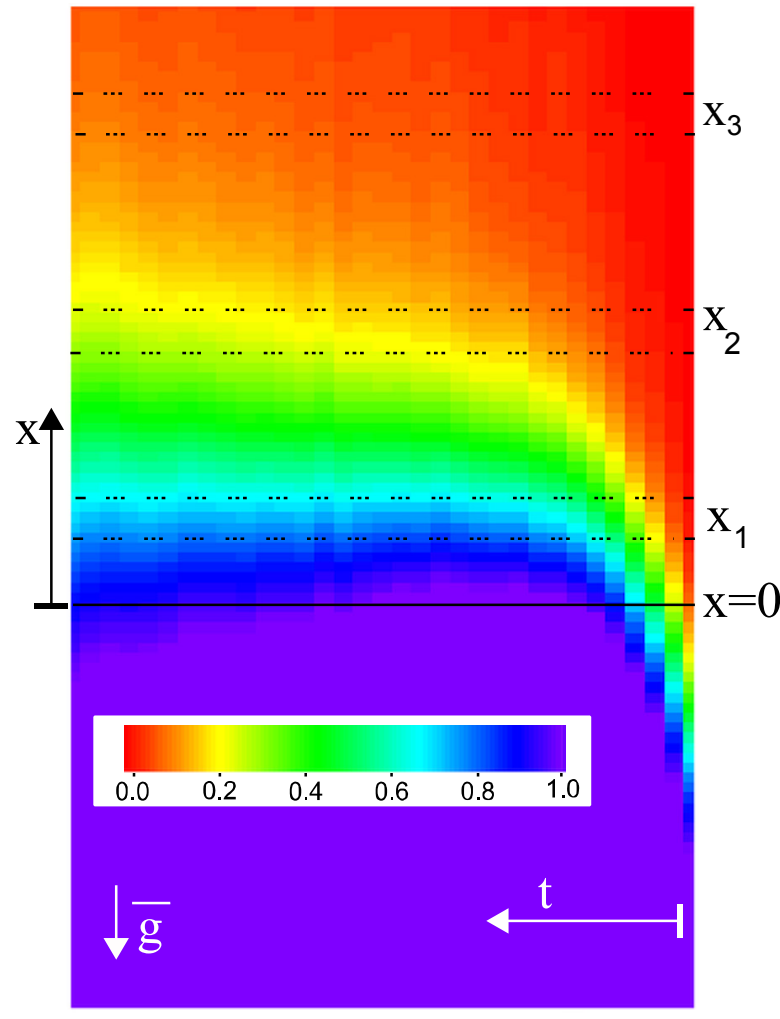

Figure 3. Spatiotemporal diagram of $C^{\prime}(x, t)$ for $\phi=3 \%$. The monitoring distances: $x_{I}=1 \mathrm{~mm}, x_{2}=2.5 \mathrm{~mm}$ and $x_{3}=4 \mathrm{~mm}$ are indicated.

\section{Results and discussion}

Fig. 4 shows the time variation of the normalized concentration $C^{\prime}(t)$ at the monitoring distance $x_{l}=1 \mathrm{~mm}$ for all the values of $\phi$ studied.

A marked change between $\phi<3 \%$ and $\phi \geq 3 \%$ is observed, in line with the onset of strong fingering regime observed in Fig. 2. The time for which this asymptotic value is attained decreases with $\phi$.

The inset shows the same variation, for $\phi=3 \%$, at the different monitoring distances $x_{\mathrm{i}}$.

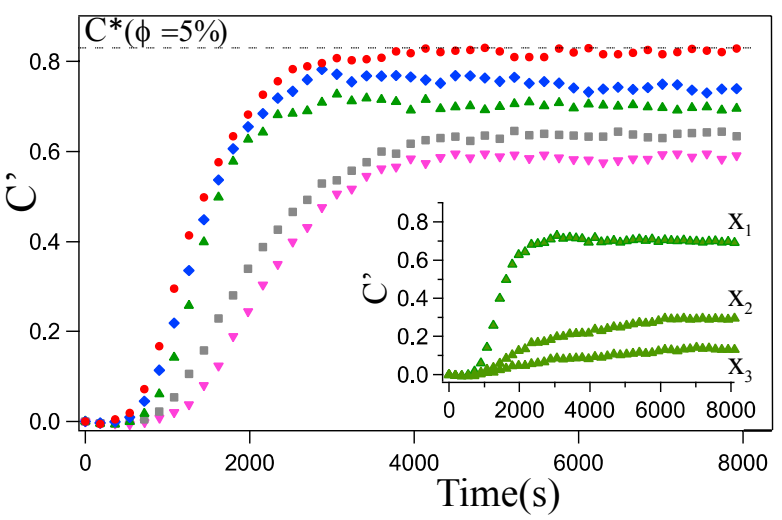

Figure 4. Time variation of the normalized concentration $C$, at $x_{I}=1 \mathrm{~mm}(\nabla: \phi=1 \%, \square: \phi=2 \%, \Delta: \phi=3 \%, \diamond: \phi=4 \%$, $\bullet: \phi=5 \%$ ). The dashed line illustrates the measurement of the asymptotic $C^{\prime}$ value $\equiv C^{*}$. Inset shows the time variation of $C^{\text {' }}$ for $\phi=3 \%(\Delta)$, at $x_{1}=1 \mathrm{~mm}$ (upper curve), $x_{2}=2.5 \mathrm{~mm}$ (middle curve) and $x_{3}=4 \mathrm{~mm}$ (lower curve).
In all cases, the concentration reaches an asymptotic value $C^{*}$, that increases with $\phi$, and decreases with $x$. The time for which this asymptotic value is attained increases with $x$.

Fig. 5 shows the variation of $C^{*}$ as a function of $\phi$, at $x_{1}=1 \mathrm{~mm}, x_{2}=2.5 \mathrm{~mm}$ and $x_{3}=4 \mathrm{~mm}$ (from top to bottom). At each monitoring position, a rather linear increase of $C^{*}$ with $\phi$ is observed.

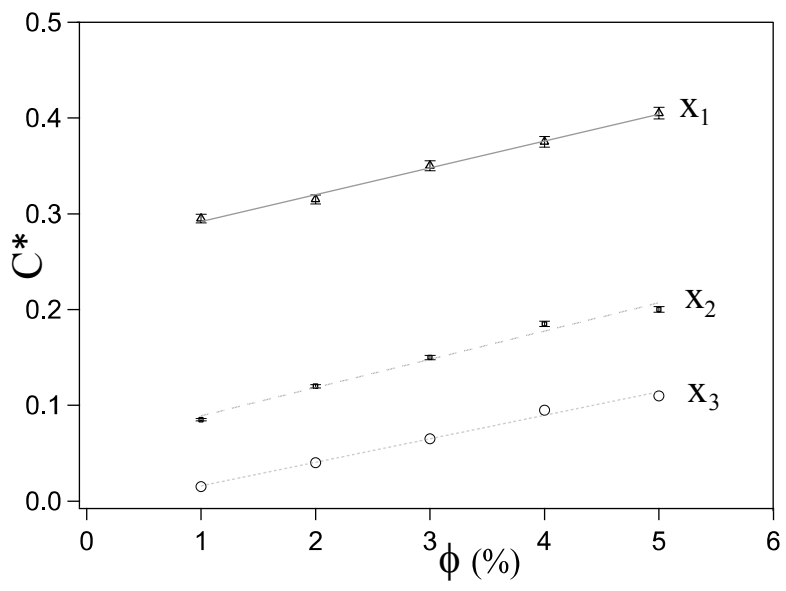

Figure 5. Asymptotic concentrations $C^{*}$ as a function of the solid fraction $\phi\left(\Delta: x_{1}=1 \mathrm{~mm}, \square: x_{2}=2.5 \mathrm{~mm}, \circ: x_{3}=4 \mathrm{~mm}\right)$. The solid, dashed, and dotted lines are linear fits over the data points for $x_{1}, x_{2}$ and $x_{3}$ respectively.

This linear behaviour might seem surprising, given the fact that, for $\phi \geq 3 \%$, the fingering becomes much stronger (Fig. 2). However, it can be observed that, for $\phi<3 \%$, there are more fingers (while relatively smaller), so the transport of the solute is likely to be more homogeneous in the $y$-direction. For long times, as the smaller fingers develop, this might generate a partial compensation in $C^{*}$. On the other hand, $C^{*}$ decreases as $x$ increases (this can be observed also in Fig. 4).

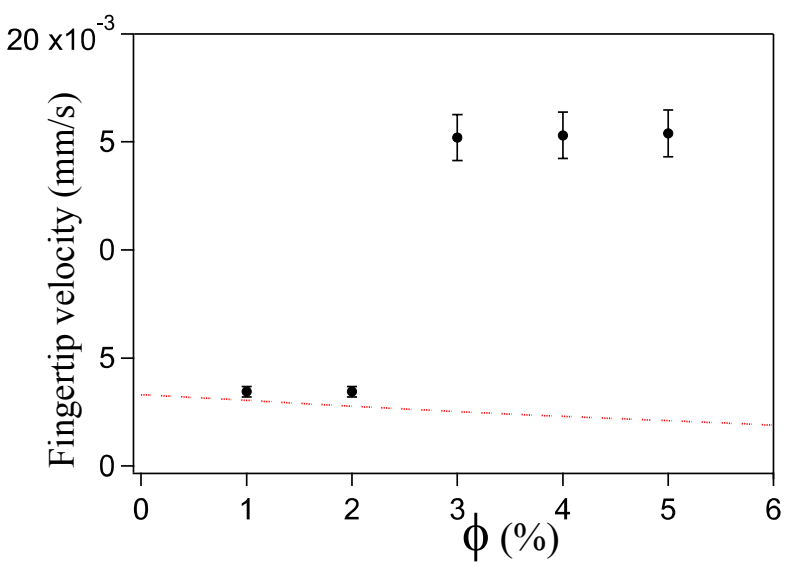

Figure 6. (•): Fingertip upwards characteristic velocity as calculated from the iso-concentration curves of Fig. 2 over different fingers at $t=1440 \mathrm{~s}$. The dashed line is the prediction of Richardson-Zaki [15] for slow buoyant displacement in a narrow vessel using our experimental data.

From the iso-concentration fronts (as that shown in inset of Fig. 2 (c)), a characteristic upwards fingertip velocity is estimated, by performing an average over 
different fingers at $\mathrm{t}=1440 \mathrm{~s}$, an early stage of the instability.

Fig. 6 shows such a velocity, and also the theoretical prediction of Richardson-Zaki for slow buoyant suspension displacement in a narrow vessel [15].

Integrating the concentration over the volume for which $x>0$, the solute mass discharge and relative mass discharge across the initial interface is calculated. Fig. 7 shows their time variation.

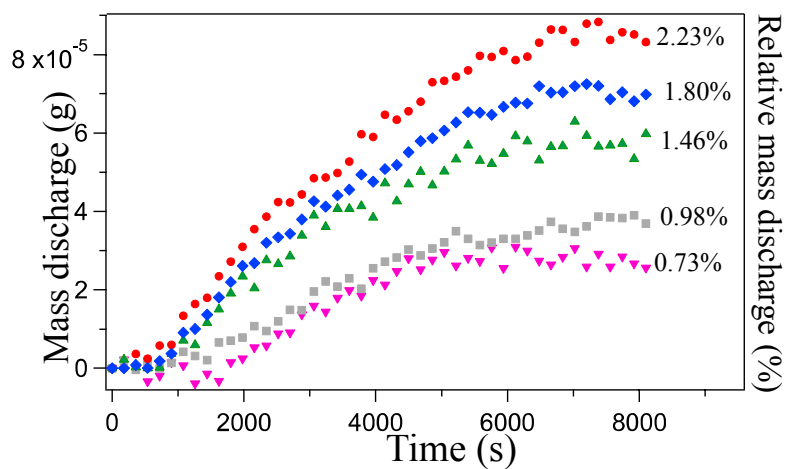

Figure 7. Solute mass discharge and relative mass discharge across the initial interface as a function of time. $(\nabla: \phi=1 \%$, 口: $\phi=2 \%, \Delta: \phi=3 \%, \bullet: \phi=4 \%, \bullet: \phi=5 \%)$.

The relative mass discharge attains $2.23 \%$ for $\phi=$ $5 \%$, indicating that the mechanism driving the transport is not efficient. However, in certain situations related to the subsurface storage of hazardous substances, this percentage is not negligible.

\section{Discussion and conclusions}

We have studied the transport of a solute by buoyant suspended particles in a Hele-Shaw cell in the absence of net flow.

The rapid increase of $C^{\prime}$ for early times, the subsequent slow-down, and the asymptotic behaviour, are strongly correlated in time with the onset, development and vanishing of the fingering instability. The same holds for the mass discharge.

If the suspension behaved as a homogeneous fluid, the initial condition would be stable (the mean density of the coloured suspension, situated below, is higher than that of the transparent fluid, because $\phi$ is small). It is then likely that the triggering and development of the instability is related to local fluctuations in the number density of the particles approaching the interface due to their early buoyant displacement. This is supported by the fact that the velocity of the fingertips for $\phi \geq 3 \%$ is 3 4 times greater than the characteristic buoyant velocity of homogeneously-distributed particles.

The evidence suggests a mechanism similar to settling-driven convection $[3,16]$ (also called convective sedimentation) as the driver of the transport. This type of instability has been observed in geosciences, for example, for the sedimentation of clay and silt fines [17], even for low values of $\phi$. Some similarities with the particle-driven Rayleigh Taylor instability [2] exist, but, in our view, it would require higher values of $\phi$ for the weak density contrasts used in this work.
At the late stage of the instability, once the fingers disappear, merging with the surrounding fluid that contains a lower density of particles (and of colorant), the transport of the solute stalls. We remark that at this stage (typically at $t \sim 8000 \mathrm{~s}$ ), particles are still rising (the last particles will reach the position of the initial interface at $t \sim 20000 \mathrm{~s}$ ), but seemingly don't drive the transport of the solute.

We conclude that transport is driven by the instability and, even if triggered by the buoyant displacement of the particles only, requires the convection of a macroscopic portion of the suspension.

The authors would like to thank A. D'Onofrio, M. Piva, G. Drazer, J.P. Hulin, D. Salin, J.C. Géminard and G. Gauthier for fruitful discussions, and J. Lamparte for his collaboration in the construction of the experimental device. Support was received from PICT-2013-2584 and PIP CONICET $11220130100230 \mathrm{CO}$.

\section{References}

[1] P.N. Segrè, E. Herbolzheimer, P.M. Chaikin, Phys. Rev. Lett. 79, 2574 (1997)

[2] C. Völtz, W. Pesch, I. Rehberg, Phys. Rev. E 65 011404 (2001)

[3] D. Hoyal, M. Bursik, J. F. Atkinson, Mar. Geol., 159, 205-220 (1999)

[4] N. Massei, M. Lacroix, H. Q., Wang, J.P. Dupont, J. Contam. Hydrol. 57, 21-39 (2002)

[5] I.M. Griffiths, H. A. Stone, EPL, 97, 5 (2012)

[6] A. Boschan, M. A. Aguirre, G. Gauthier, Soft Matter, 11, 17 (2015)

[7] G.M. Nirmal, A. Ramachandran, Soft Matter, 12, 38 (2016)

[8] T. Duman, R. Holtzman, U. Shavit, Int. J. Multiph. Flow, 106, 220-227 (2018)

[9] S. Ruhl, C. Thompson, A. Queiros, S. Widdicombe, ICES J. Mar. Sci., 77, 5 (2020)

[10] F. Blanchette, W. Douandju, S.M. Montroy, Phys. Fluids, 22, 123304 (2010)

[11] A. Boschan, B. Ocampo, M. Annichini, G. Gauthier, Phys. Fluids. 28, 063301 (2016)

[12] M.S. Plesset, C.G. Whipple, Phys. Fluids, 17, 1 (1974)

[13] A. Boschan, H. Auradou, R. Chertcoff, I. Ippolito, J.P. Hulin, Water Resour. Res., 43, W03438 (2007)

[14] G. Cao, R. Qin, Y. Wu, Environ. Fluid. Mech., 18, 465-486 (2018)

[15] J.F. Richardson, W.N. Zaki, Trans. Inst. Chem. Eng. 32, 35-53 (1954)

[16] Y. Shao, C. Hung, Y. Chou, J. Fluid. Mech., 824, 513-549 (2017)

[17] X. Yu, T.J. Hsu, S. Balachandar, J. Geophys. Res. Oceans, 119, 8141-8161 (2014) 